This is an electronic reprint of the original article. This reprint may differ from the original in pagination and typographic detail.

Author(s): Zhang, Di; Chang, Zheng; Zolotukhin, Mikhail; Hämäläinen, Timo

Title: $\quad$ Energy Efficient Resource Allocation in Heterogenous Software Defined Network : A Reverse Combinatorial Auction Approach

Year: $\quad 2015$

Version:

Please cite the original version:

Zhang, D., Chang, Z., Zolotukhin, M., \& Hämäläinen, T. (2015). Energy Efficient Resource Allocation in Heterogenous Software Defined Network : A Reverse Combinatorial Auction Approach. In Proceedings of the 4th IEEE/CIC International Conference on Communications in China (ICCC'2015). Symposium on Signal Processing for Communications (pp. 739-744). IEEE. https://doi.org/10.1109/ICCChina.2015.7448686

All material supplied via JYX is protected by copyright and other intellectual property rights, and duplication or sale of all or part of any of the repository collections is not permitted, except that material may be duplicated by you for your research use or educational purposes in electronic or print form. You must obtain permission for any other use. Electronic or print copies may not be offered, whether for sale or otherwise to anyone who is not an authorised user. 


\title{
Energy Efficient Resource Allocation in Heterogeneous Software Defined Network: A Reverse Combinatorial Auction Approach
}

\author{
Di Zhang, Zheng Chang, Mikhail Zolotukhin and Timo Hämäläinen \\ Department of Mathematical Information Technology, University of Jyväskylä, P.O.Box 35, FIN-40014 Jyväskylä, Finland \\ Email: zhdizhan@student.jyu.fi, \{zheng.chang, mikhail.m.zolotukhin, timo.t.hamalainen\}@jyu.fi
}

\begin{abstract}
In this paper, resource allocation for energy efficiency in heterogeneous Software Defined Network (SDN) with multiple network service providers (NSPs) is studied. The considered problem is modeled as a reverse combinatorial auction game, which takes different quality of service $(\mathrm{QoS})$ requirements into account. The heterogeneous network selection associated with power allocation problem is optimized by maximizing the energy efficiency of data transmission. By exploiting the properties of fractional programming, the resulting non-convex Winner Determination Problem (WDP) is transformed into an equivalent subtractive convex optimization problem. The proposed reverse combinatorial auction game is proved to be strategy-proof with low computing complexity. Simulation results illustrate that with SDN controller, the proposed iterative ascending price algorithm converges in a small number of iterations and demonstrates the trade-off between energy efficiency and heterogeneous QoS requirement, especially ensures high fairness among different network service providers.
\end{abstract}

\section{INTRODUCTION}

Future 5G network is expected to be heterogeneous in nature for providing gigabits data transmission for a large number of mobile devices [1]. Software Defined Network $(\mathrm{SDN})$ is proposed as the future of $5 \mathrm{G}$ mobile networks by leveraging the programmability advantages in the separation of control-data plane [2]. Within this architecture, the network services provider (NSP) is no longer limited to the traditional operator that owns the infrastructure, but can also contain the virtual operators that are able to rent the infrastructure for providing services [3], [4]. There is no doubt that the radio access network will become much more heterogeneous, complex and denser, which calls for a new resource allocation mechanism to cope with the personal QoS requirement and system energy efficiency [5]. Meanwhile, widely deploying more and more access points to meet the increasing data demand inevitably increase the power consumption. Therefore, designing an energy efficient architecture to cope with the mixed usage of cells with diverse sizes and numbers of access points for a heterogeneous SDN access network is attracting more and more research attentions.

In this paper, we study how to efficiently coordinate the limited resource for different NSPs to maximize the profits and obtain higher fairness within SDN-based heterogeneous architecture. Most of the existing resource allocation schemes used in the traditional architectures are not appropriate for the heterogeneous SDN networks [6]-[8] due to the following reasons: 1) algorithm in the control plane lacks the availability of user equipment (UE) information (e.g., relative distance between the base stations and users), which requires extensive information exchange; 2) the lack of considering NSPs, as they are selfish and only focus on their own profit, which calls for a contiguous design of novel resource allocation scheme.

Motivated by the aforementioned observations, we study the SDN architecture with reverse combinatorial auction algorithm installed to address the energy efficient resource allocation problem by considering the competitive and fairness among multiple NSPs. Auction theory is introduced to provide an interdisciplinary technology for radio resource allocation (e.g., sub-carriers, time slots, and transmit power levels) in the wireless systems [9]. By using various auction approaches, such radio resources are efficiently allocated among users and providers in the cellular system [10]. The auction based method has been applied for the cognitive radio and mobile ad-hoc systems [11], [12] massively, but is less discussed in wireless cellular systems. In [12], the authors consider a twostage resource allocation scheme with combinatorial auction in spectrum sharing problem, however, it does not consider more specific information about primary and secondary spectrum users. In [13], the authors propose one sequential single-item auction, where each user submits a bid based on the marginal increase in the data rate, but they did not consider multiple network providers.

In this work, the proposed reverse combinatorial auction based resource allocation scheme aims at maximizing energy efficiency while taking different QoS constraints (maximum power requirement and user demand) into account. The contributions of this work can be summarized as follows. First, we formulate the energy efficiency resource allocation problem among different NSPs as a reverse combinatorial auction game by considering different QoS requirement. The resulting nonconvex WDP problem in fractional form is transformed into an equivalent subtractive optimization one by exploiting the properties of fractional programming. After that, this problem is solved by an iterative ascending price auction (IAPA) algorithm, which is proved to be strategy-proof. Simulation results illustrate that, with SDN controller, the proposed algorithm shows good results in terms of convergence speed and can offer high fairness allocation among NSPs. Moreover, it is also proved to be robust with QoS requirement changing. 


\section{System Model AND WinNer Determination PROBLEM FORMULATION}

\section{A. System Model}

We consider a heterogeneous SDN within one denser district, which consists of $J$ Network Service Providers (NSPs) $(\mathcal{J}=\{1, \ldots, J\})$ and $I$ user equipments (UEs) $(\mathcal{I}=$ $\{1, \ldots, I\})$. The SDN controller acts as a central controller, which takes charge of all resource management related algorithms in this geographical area. Each NSP is assumed to operate on different licensed spectrum and is further assumed to observe only one base station (BS) with QoS requirement of maximum total power consumption constraint, $\mathcal{P}_{j, \max }=$ $\left[P_{1, \max }, \ldots, P_{J, \max }\right]$ separately (without loss of generality, we use NSP and BS interchangeablely). UEs are assumed to be uniformly distributed in the geographical area with individual requirement of data rate $\mathcal{R}_{i, \text { min }}$. NSPs obtain heterogeneous resource of both channels and power constraints.

In this paper, we consider the problem of how to allocate the heterogeneous resource in order to achieve maximum energy efficiency (higher system throughput and lower system power consumption) and satisfy the QoS requirement. The energy efficient resource allocation (EE-RA) problem is formulated as a Reverse Combinatorial Auction (R-CA) Game. We introduce a non-profit central entity in this game, which is responsible for running the resource allocation auction game, called resource broker. The resource broker with algorithms for solving the auction game is installed on the SDN controller. The NSPs who observe resource (BSs) and money constraint $\left(\mathcal{P}_{j, \max }=\left[P_{1, \max }, \ldots, P_{J, \max }\right]\right)$ act as bidders and bid for the business with UEs. The UEs which want to join the network act as sellers or items with price requirement of ( $\left.\mathcal{R}_{i, \min }=\left[R_{1, \min }, \ldots, R_{I, \min }\right]\right)$.

\section{B. Channel Efficiency}

In this paper, the channel is modeled as the Rayleigh fading channel [8]. We define $\mathrm{SNR}_{j i}$ as the signal to noise ratio (SNR) from NSP $j \in \mathcal{J}$ to $\mathrm{UE} i \in \mathcal{I}$,

$$
\mathrm{SNR}_{j i}=\frac{p_{j i}\left|H_{j i}^{2}\right|}{N_{0}},
$$

where $p_{j i}$ is the allocated power from BS $j$ to UE $i, N_{0}$ is the additive white Gaussian noise (AWGN) at the receivers with one-sided power spectral density. $\left|H_{j i}^{2}\right|$ is the channel gain, where $\left|H_{j i}^{2}\right|=d_{j i}^{-\alpha}\left|h_{j i}\right|^{2}$. $\left|h_{j i}\right|$ is the complex Gaussian channel coefficient which obeys the distribution $\mathcal{C N}(0,1), d_{j i}$ is the relative distance from $\mathrm{BS} j$ to $\mathrm{UE} i$, and $\alpha$ is the free space path-loss exponent.

We calculate the channel rate according to the SNR between BS $j$ and UE $i . r_{j i}$ is defined as the data rate received by UE $i$ from BS $j$ :

$$
r_{j i}=\log _{2}\left(1+\mathrm{SNR}_{j i}\right)=\log _{2}\left(1+\frac{p_{j i}\left|h_{j i}\right|^{2}}{d_{j i}^{\alpha} N_{0}}\right),
$$

from which we can infer that, the channel rate $r_{j i}$ is related to the transmission power $p$ between transmitter $j$ and receiver $i$, so we have $r(j, i, p)=r_{j i}$, and $\rho(j, i, p)=p_{j i}$, where $\rho$ stands for that it is the function of $p$.

We define Channel Efficiency as the ratio between channel rate $r(j, i, p)$ and channel transmission power $\rho(j, i, p)$ :

$$
C_{j i}^{e f f} \triangleq \frac{r_{j i}}{p_{j i}}=\frac{\log _{2}\left(1+\frac{p_{j i} h_{j i}^{2}}{d_{j i}^{\alpha} N_{0}}\right)}{p_{j i}}, C^{e f f}(j, i, p)=\frac{r(j, i, p)}{\rho(j, i, p)},
$$

where $C_{j i}^{e f f}$ is the calculation form of the Channel Efficiency, and $C^{e f f}(j, i, p)$ means that Channel Efficiency is a function of power $p$.

\section{Bidding Strategy of Reverse Combinatorial Auction}

In this section, we define that, the bidding strategy composes two parts $\left(\mathcal{S}_{j}, \mathcal{B}_{j}\right)$ for every bidder. The first part is the bidding bundle, which is the subset $\mathcal{S}_{j}$ of the whole items $\mathcal{I}$. The second part is the associated bidding price $\mathcal{B}_{j}$, which will directly affect the final winner determination. The detailed bidding strategy is described as follows.

1) Bidding Bundle Expression: We define set $\mathcal{S}(\mathcal{S} \subseteq \mathcal{I})$ as a bundle of variables representing the business connection between NSP and UE. It can range from $\emptyset$ to $\mathcal{I}$, so for every bidder, there are $2^{I}$ such bundles to be calculated and chosen.

2) Asymmetric Valuation of Bidding Bundle: In order to execute this resource auction game more realistically, we define the valuation of bundle $\mathcal{S}$ as $V_{j \mathcal{S}}$, which is also the ratio between channel rate and channel transmission power (see the definition in Section II-B), where $V_{j \mathcal{S}} \triangleq C_{j \mathcal{S}}^{\text {eff }} \triangleq$ $\frac{\sum_{i \in \mathcal{S}} r_{j i}}{\sum_{i \in \mathcal{S}} p_{j i}}, \forall j \in \mathcal{J}$. This definition also expresses the asymmetric property of the valuation, which means when bidder $j$ changes, the valuation of the same bundle $\mathcal{S}$ also changes. With the same definition method in (3), we have

$$
V(j, \mathcal{S}, p)=\frac{r(j, \mathcal{S}, p)}{\rho(j, \mathcal{S}, p)}=\frac{\sum_{i \in \mathcal{S}} r(j, i, p)}{\sum_{i \in \mathcal{S}} \rho(j, i, p)}, \forall j \in \mathcal{J},
$$

which means that the valuation of bundle $\mathcal{S}$ depends on not only the transmitter $j$, but also the allocated power $\rho$.

3) Bidding Price: To achieve incentive compatibility, the auction mechanism should be designed to guarantee that, the dominant bidding strategies of bidders are the truthful bidding strategies. So in this paper we consider Vickrey-Clarke-Groves auction [11]. VCG is a type of truthful auction, which means the bidder will bid the bundle according to its real valuation, no matter what other bidders' bidding strategy are:

$$
B_{j \mathcal{S}}=V_{j \mathcal{S}}, B(j, \mathcal{S}, p)=V(j, \mathcal{S}, p), \forall j \in \mathcal{J} .
$$

Now the bidding strategy of bidder $j$ would be pair $\left(\mathcal{S}_{j}, \mathcal{B}_{j}\right)$. Note that the whole bidding strategy space of bidder $j$ is $\mathcal{C}_{\mathcal{S}}(\mathcal{I}) \times \mathbb{R}^{+}$, where $\mathcal{C}_{\mathcal{S}}(\mathcal{I})$ means the combination of choosing $\mathcal{S}$ from set $\mathcal{I}$.

4) Pay Price: The cost of bidder $j$ should pay for item $i$ is defined as pay price and expressed as $Q_{j \mathcal{S}}($ also as $Q(j, \mathcal{S}, p)$ ). In order to extend the combinatorial resource auction to a strategy-proof auction mechanism, we define the pay price observing the properties of non-linearity and anonymity, where if $\exists \mathcal{S}=\mathcal{S}_{1} \cup \mathcal{S}_{2}$, then it does not mean $Q_{j \mathcal{S}}=Q_{j \mathcal{S}_{1}}+Q_{j \mathcal{S}_{2}}$ and if $\exists j \neq j^{\prime}$, then it does not mean $Q_{j \mathcal{S}}=Q_{j^{\prime} \mathcal{S}_{1}}$. 
5) Bidder Utility: During the auction, each NSP obtains a gain by offering connection with a bundle of UEs. The difference between the valuation of bidding bundle and the pay price is what bidder $j$ can obtain finally. We define the gain of bidder $j$ as the utility, $U_{j \mathcal{S}}$ :

$$
U_{j \mathcal{S}}= \begin{cases}V_{j \mathcal{S}}-Q_{j \mathcal{S}}, & \text { if bidder } \mathrm{j} \text { wins bundle } \mathcal{S} \\ 0, & \text { otherwise },\end{cases}
$$

where $U_{j \mathcal{S}}$ is the calculated payoff of bidder $j$ for bundle $\mathcal{S}$. If bidder $j$ wins, the utility will be calculated according to definition, and can be expressed as $U(j, \mathcal{S}, p)$ according to (4) and (5); if bidder $j$ loses, SDN controller will charge nothing for the bidding.

\section{Winner Determination Problem}

The problem of identifying which set of bids to be accepted is usually been defined as the Winner Determination Problem (WDP). According to WDP, the objective is the social welfare maximization, which is the overall gain of both bidders and sellers (or items).

1) Bidding Language and Decision Variables: We refer to the non-exclusive bundle-bids as the bidding language, which is also can expressed as XOR language. Within this mode, each bidder can submit an arbitrary number of pairs $\left(\mathcal{S}_{j}, \mathcal{B}_{j}\right)$, however, at most one of these bids can win finally. We define $x_{j \mathcal{S}}$ as the decision variable, which means for certain bundle $\mathcal{S}$, whether bidder $j$ wins or not:

$$
x_{j \mathcal{S}}= \begin{cases}1, & \text { if bidder } \mathrm{j} \text { wins bundle } \mathcal{S} \\ 0, & \text { otherwise. }\end{cases}
$$

$x_{j \mathcal{S}}$ expresses the relationship between bundle $\mathcal{S}$ and bidder $j$ with decision space of $J \times 2^{I}$, which has the same meaning in section II-C1. With decision variable $x_{j \mathcal{S}}$, we rewrite many functions above as follows: bundle $\mathcal{S}$ 's total rate can be rewritten as $r(x, p)$, total power consumption can be rewritten as $\rho(x, p)$. Therefore, the valuation in (4) can be rewritten as $V(x, p)=\frac{r(x, p)}{\rho(x, p)}$.

2) Social Welfare: We propose Theorem 1 for the definition of social welfare.

Theorem 1: The social welfare of this reverse combinatorial auction is the system energy efficiency $\gamma$, which is the proportion of system throughput $R(x, p)$ and system power consumption $P(x, p)$.

Proof: For bidder $j$, the welfare is the utility of bidding strategy $U(x, p)$, and for seller $i$, the welfare is the gain that bidder $j$ has offered $Q(x, p)$. We define $A(\mathcal{X})$ as the sellers' total welfare and $B(\mathcal{X})$ as the bidders' total welfare:

$$
\begin{aligned}
& A(\mathcal{X})+B(\mathcal{X})=\sum_{x \in \mathcal{X}} \sum_{p \in \mathcal{P}} Q(x, p)+\sum_{x \in \mathcal{X}} \sum_{p \in P} U(x, p) \\
& =\sum_{x \in \mathcal{X}} \sum_{p \in \mathcal{P}} V(x, p),
\end{aligned}
$$

where $\sum_{x \in \mathcal{X}} \sum_{p \in \mathcal{P}} V(x, p)=\frac{\sum_{x \in \mathcal{X}} \sum_{p \in \mathcal{P}} r(x, p)}{\sum_{x \in \mathcal{X}} \sum_{p \in \mathcal{P}} \rho(x, p)}=\frac{R(x, p)}{P(x, p)}=$ $\gamma$, the social welfare is the ratio between system throughput and power consumption.
3) Formulation of WDP: We formulate the WDP as a mixed 0-1 integer non-linear programming with the social welfare maximization and QoS requirement satisfaction.

$$
\max _{x, p} \gamma=\frac{R(x, p)}{P(x, p)},
$$

$$
\begin{aligned}
\text { s.t. } C 1: x_{j \mathcal{S}}=\{0,1\}, \rho(j, i, p)>0, \forall \mathcal{S} \subseteq \mathcal{I}, \forall j \in \mathcal{J}, \\
C 2: \sum_{i \in \mathcal{I}} \rho(j, \mathcal{S}, p) \leq P_{j, \max }, \forall j \in \mathcal{J}, \\
C 3: \sum_{j \in \mathcal{J}} x_{j \mathcal{S}} \leq 1, \forall i \in \mathcal{I}, \\
C 4: \sum_{j \in \mathcal{J}} r(j, i, p) \geq R_{i, \min }, \forall i \in \mathcal{I}, \\
C 5: \sum_{\mathcal{S} \subseteq \mathcal{I}} x_{j S} \leq 1, \forall j \in \mathcal{J} .
\end{aligned}
$$

\section{ITERATIVE ASCENDING PRICE AUCTION ALGORITHM FOR ENERGY EFFICIENT RESOURCE ALLOCATION}

In this section, we propose an iterative algorithm for solving the problem in section II-D3. We first exploit the non-linear fractional WDP for converting the objective function into an equivalent subtractive one [14], upon which we successfully transform it into a convex combinatorial optimization problem.

\section{A. Problem Transformation}

Without loss of generality, we define the optimal maximum energy efficiency parameter as $\gamma^{*}$, and $\mathcal{F}$ as the set of feasible solutions of WDP problem in section II-D3:

$$
\gamma^{*}=\frac{R\left(x^{*}, p^{*}\right)}{P\left(x^{*}, p^{*}\right)}=\max _{(x, p) \in \mathcal{F}} \frac{R(x, p)}{P(x, p)} .
$$

Theorem 2: $\gamma^{*}$ is achieved if and only if

$$
\begin{aligned}
& \max _{(x, p)} R(x, p)-\gamma^{*} P(x, p) \\
& =R\left(x^{*}, p^{*}\right)-\gamma^{*} P\left(x^{*}, p^{*}\right)=0,
\end{aligned}
$$

where $\{x, p\}$ is any feasible solution of problem (9) to satisfy the constraints in (10).

Proof: The proof of Theorem 2 is presented in [14].

\section{B. Iterative ascending price auction algorithm for Energy Efficiency Maximization}

In this section, we propose an iterative ascending price auction algorithm (IAPA algorithm) for solving the transformed convex combinatorial problem in (12). Based on the famous Dinkelbach method [14], we introduce one double loop mechanism. The outer loop is mainly dealing with the optimal $\gamma$, and the inner loop is the main iterative reverse combinatorial auction game. The SDN controller will take charge of the whole algorithm, both the outer loop and inner loop. Firstly, within the outer loop, SDN controller will control the energy efficiency parameter $\gamma$ by changing the maximum iteration number $K_{\max }$ and convergence tolerance $\varepsilon$, the detailed is summarized in Algorithm 1. Secondly, within the inner loop, SDN controller will act as the resource broker 


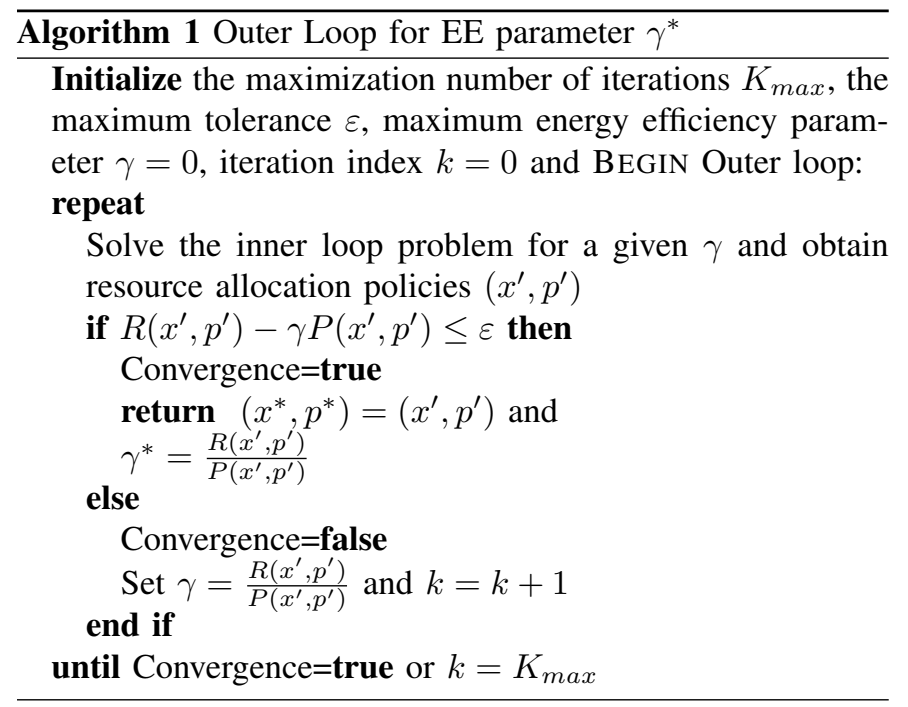

to control the whole reverse combinatorial auction game by determining an optimal allocation scheme which leads to a social optimality. In addition, the SDN controller will also calculate the payments and payoffs for bidders. The detailed is summarized in Algorithm 2.

As shown in Algorithm 1, in each outer loop iteration, we solve the following optimization problem for a given parameter $\gamma$ :

$$
\begin{gathered}
\max _{(x, p)} \mathcal{Z}(x, p)=R(x, p)-\gamma P(x, p), \\
\text { s.t. } C 1, C 2, C 3, C 4, C 5 .
\end{gathered}
$$

While the introduced inner loop iterative reverse combinatorial auction game is one kind of combinatorial optimization problem with given $\gamma$, which is hard to be solved within polynomial time [10]. We propose an IAPA algorithm according to [15], which is proved to solve the reverse combinatorial auction game efficiently. In Algorithm 2, we introduce a strategy-proof price updating mechanism, where the price is updated by a greedy mode, and once the bidder submits a bid for items or bundle, the corresponding price named hammer price is fixed, otherwise the price is increased. This IAPA algorithm is also demonstrated to guarantee both individual rationality and incentive compatibility (truthfulness) in III-C.

SDN controller controls the whole reverse combinatorial auction by changing the price updating mechanism, we define $\mathcal{Q}$ as the updating mechanism:

$$
\mathcal{Q} \leftarrow q=\frac{\min _{i \in \mathcal{I}} R_{i, \text { min }}}{\max _{j \in \mathcal{J}} P_{j, \text { max }}},
$$

where we can see, when the iteration $t$ changes, $R_{i, \min }$ and $P_{j, \max }$ will accordingly increase and decrease. The price $q_{i}$ changes in a non-monotonous way, which can also express the properties (non-linearity and anonymity) defined in section II-C4.

\section{Strategy-proof}

As the general definition, strategy-proof means reporting the true demand in each iteration auction is the best response for

\section{Algorithm 2 Inner Loop auction for RA $\left(x^{*}, p^{*}\right)$}

Input Outer Loop given $\gamma$, Initialize BS total power assignment $P_{j}=0$ and $\mathrm{BS}$ total throughput $R_{j}=0$, iteration index $t=0, \mathcal{J}, \mathcal{I}$, QoS vector $\mathcal{P}_{j, \text { max }}$ and $\mathcal{R}_{i, \text { min }}$

SDN controller Sets initial auction price $q$ and update mechanism $\mathcal{Q}$ according to (14)

while $\mathcal{I} !=0$ do

Bidding Strategy Generation

for all $j=1 \ldots J$ do

for all $i=1 \ldots I$ do

Calculate $p_{j i}=\frac{\left(2^{R_{i}}-1\right) d_{j i} N_{0}}{\left|h_{j i}\right|^{2}}$ according to (2)

\section{end for}

Sort $p_{j i}$ and $P_{j, \max } \leftarrow \max \left(p_{j i}\right)$

while $P_{j} \leq P_{j, \max }$ do

$b_{j i}=R_{i}-q \times p_{j i}$

if $b_{j i}>0$ then

Bidding Set $S_{j} \leftarrow i$ and Bidding price $B_{j} \leftarrow b_{j i}$ $P_{j}=P_{j}+p_{j i}$

end if

end while

Submit Bidding Strategy $\left\{S_{j}, B_{j}\right\}$ for every $j \in \mathcal{J}$

\section{end for}

\section{SDN controller determines winner}

for all $i=1 \ldots I$ do

Sort $b_{j i}$ with descending mechanism, and find index $j^{\prime}$ with $\max \left(b_{j i}\right)$

$$
\begin{aligned}
& \text { if } b_{j^{\prime} i} \geq\left(R_{i}-\gamma p_{j^{\prime} i}\right) \text { then } \\
& \quad x_{j^{\prime} i}=1, x^{*} \leftarrow x_{j^{\prime} i}, p^{*} \leftarrow p_{j^{\prime} i}, P_{j}^{\prime}=P_{j}^{\prime}+p_{j^{\prime} i} \\
& \quad \mathcal{I}=\mathcal{I}-x^{*}, P_{j^{\prime}, \text { max }}=P_{j^{\prime}, \text { max }}-p_{j^{\prime} i}, R_{j}^{\prime}=R_{j}^{\prime}+R_{i} \\
& \text { end if }
\end{aligned}
$$

\section{end for}

Set $\mathrm{t}=\mathrm{t}+1$, and Update $\mathcal{Q}$ end while

every bidder. We demonstrate the proposed IAPA algorithm ensuring the proposed bidding strategy in each iteration is the best response of every bidder $j$.

Theorem 3: The iterative ascending price resource allocation algorithm within inner loop is strategy-proof.

Proof: We consider two cases for bidding, where the strategy $(\mathcal{S}, \mathcal{B})$ of bidder $j$ has true valuation $V(j, \mathcal{S}, p)$. 1) During the beginning round, the utility for bidder $j$ is $V(j, \mathcal{S}, p)-q>0$, if $j$ quit this round and wait for another chance, it will lose the bundle which can maximize its final valuation; 2) During the following round, as the price $q$ increases, the utility for bidder $j$ will be $V(j, \mathcal{S}, p)-q<0$, if $j$ bids and finally wins this bundle $\mathcal{S}$, it will obviously get a negative surplus for the final energy efficiency valuation.

From the above analysis, we can conclude that the optimal bidding strategy for bidder $j$ is to bid with its true valuation, otherwise, it will impair its own revenue and finally reduce the system energy efficiency, i.e., the proposed algorithm is strategy-proof. 


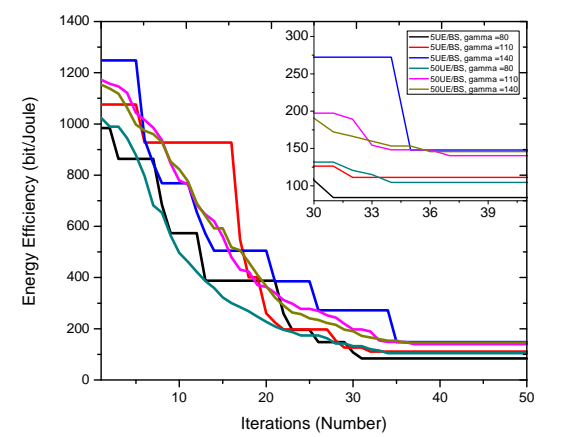

Fig. 1. Convergence speed of inner loop with different UE density and outer loop EE parameter $\gamma$ setting, for $\mathcal{P}_{j, \text { max }}=46 \mathrm{dBm}(\forall j \in \mathcal{J})$

\section{Complexity}

Traditional WDP in fact is NP-hard problem [10], especially with the non-convex objective and mixed integer decision variable. We demonstrate that the proposed iterative ascending price auction algorithm reduce the complexity of computational space significantly.

To obtain an optimal solution, an exhaustive search $(j, \mathcal{S}, p)$ is needed with complexity of $\mathcal{O}\left(J \times 2^{I+1}\right)$ for the original problem (9). While in the proposed IAPA algorithm, the computing space during every iteration for submitting biding strategy is $\mathcal{O}\left(J\left(I^{2}+2 I\right)\right.$, where $I^{2}$ is the complexity of SDN controller executed bubble sorting algorithm. For deciding the round winner, the computing sorting mechanism's complexity is $\mathcal{O}\left(I\left(J^{2}+J\right)\right)$. If the total number of inner loop is $t$, and outer loop maximization iteration is $K_{\max }$, the computing space of the proposed algorithm is:

$$
\begin{aligned}
& \mathcal{O}\left(\left(t K_{\max }\right) J\left(I^{2}+2 I\right)\right)+\mathcal{O}\left(\left(t K_{\max }\right) I\left(J^{2}+J\right)\right) \\
& =\mathcal{O}\left(\left(t K_{\max }\right) I J(I+J+3)\right)<\mathcal{O}\left(J \times 2^{I+1}\right),
\end{aligned}
$$

where we can conclude that the IAPA algorithm can reduce the complexity significantly compared to the original one (9).

\section{Performance Evaluation}

In this section, we provide the simulation results to illustrate the performances of the proposed reverse IAPA algorithm. The considered SDN heterogeneous cellular network operates within one dense geographical area with radius of $0.5 \mathrm{~km}$ with 3 NSPs. SDN controller will take charge of the whole radio resource management algorithms as well as signalling process. The UEs are randomly distributed within this area with mimimum data rate requirement $\left(\mathcal{R}_{i, m i n}=10 \mathrm{bit} / \mathrm{s} / \mathrm{Hz}\right)$. We vary the UE density in the simulation from $5 / \mathrm{BS}$ to $50 / \mathrm{BS}$. The considered path loss factor is $\alpha=4$, AWGN is $N_{0}=-131 \mathrm{dBm}$ and $h_{j i}$ obeys $\mathcal{C N}(0,1)$.

In Fig. 1, the convergence speed of the proposed IAPA algorithm with different UE density and different outer loop EE parameter $\gamma$ setting is illustrated. Generally speaking, the proposed IAPA algorithm in inner loop converges fast, even for high UE density (50/BS) and high EE objective $(\gamma=130)$

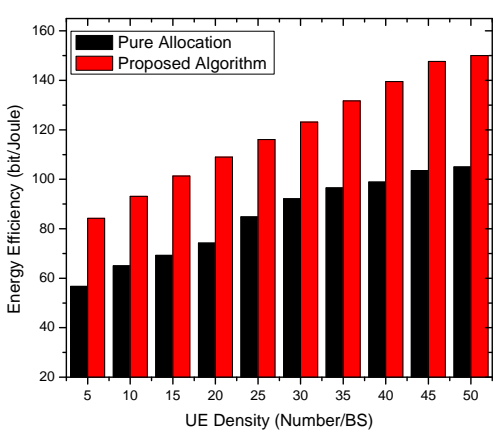

Fig. 2. Comparison of Energy efficiency between proposed IAPA algorithm and PA, for UE density $=50 / \mathrm{BS}, \mathcal{P}_{j, \max }=46 \mathrm{dBm}(\forall j \in \mathcal{J})$

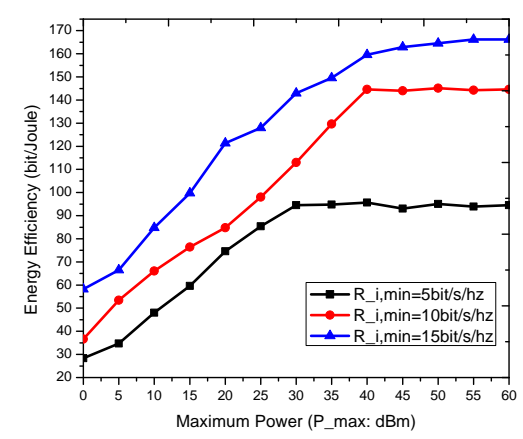

Fig. 3. Energy Efficiency versus maximum transmit power $\mathcal{P}_{j, \max }(\forall j \in \mathcal{J})$ and different $\mathcal{R}_{i, \min }$ setting $(\forall i \in \mathcal{I})$, for UE density $=5 / \mathrm{BS}$

scenarios. Within the zooming-in figure in the upper-rightcorner in Fig. 1, we can see that, the calculated EE objective $\gamma^{*}=148$ in the inner loop is better than the objective setting $\gamma=130$ of the outer loop. Besides, we can also conclude that, for certain $\gamma$, when UE density increases, the convergence speed is impaired a little bit, but the performance of EE will be improved. While for certain UE density, when EE objective setting $\gamma$ increases, the performance will be heighten obviously.

In Fig. 2, we show the system energy efficiency performance of our proposed IAPA algorithm. For comparison purpose, the pure allocation (PA) is also simulated as the benchmark, which iteratively selects the unallocated UE with minimum data rate demand and assign it to NSPs regardless of bidding price. From which, we can figure out that EE performance is strictly higher than PA scheme, it is because in our algorithm, bidders with higher valuation and bidding price would receive more attention from SDN controller according to (4).

Fig. 3 illustrates the EE performance versus heterogeneous QoS requirement. It can be observed that when the maximum transmit power is large enough, e.g., $\mathcal{P}_{j, \max }>40 \mathrm{dBm}$, the energy efficiency approaches to a constant value, since the SDN controller is not willing to consume more power for serving the $\mathcal{R}_{i, \text { min }}$ QoS requirement. Besides, the speed for 


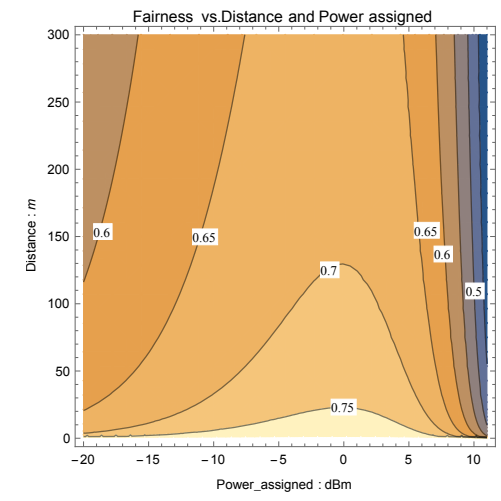

Fig. 4. Fairness of WDP between different network service providers, for $\mathcal{P}_{j, \max }=46 \mathrm{dBm}(\forall j \in \mathcal{J})$, for UE density $=5 / \mathrm{BS}$

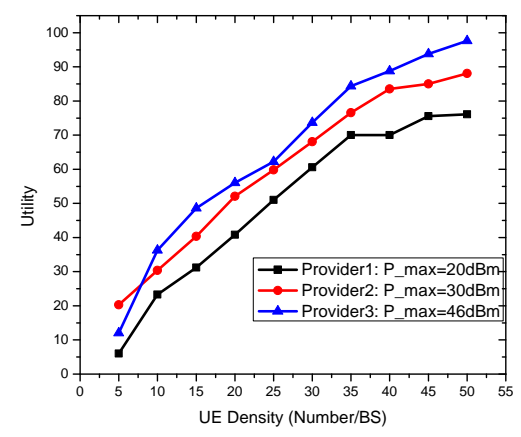

Fig. 5. Utility of different network service providers vs. different UE density

approaching stable EE is faster when $\mathcal{R}_{i, \min }$ is smaller (case of $\mathcal{R}_{i, \min }=5 \mathrm{bit} / \mathrm{s} / \mathrm{Hz}$ ), but the energy efficiency performance is strictly higher when data rate requirement is higher (case of $\mathcal{R}_{i, \min }=15 \mathrm{bit} / \mathrm{s} / \mathrm{Hz}$ ). This is due to the fact that in the proposed algorithm, SDN controller tends to use the minimum power for satisfying minimum data rate requirement, so when $\mathcal{R}_{i, \min }$ increases, the power allocated will be increased accordingly even though there is still retained power left.

In Fig. 4, we illustrate the fairness among different NSPs with a commonly used metric Jain fair index [10]. It shows that, the fairness is not only depends on the relative distance $d_{j i}$, but also the power assigned by bidder $j$. For a certain power, expansion of distance leads to the decrement of fairness. While for certain distance $d_{j i}$, when power assigned is lower than $0 \mathrm{dBm}$, the fairness will be increased with power, but when power assigned is higher than $0 \mathrm{dBm}$, the throughput is increased not much with power, it is because when throughput improves obviously (2), the EE (3) is increased accordingly, which leads to higher energy efficiency and so the higher fairness.

Fig. 5 illustrates the utility among different NSPs versus different UE density. Together with the observation in Fig. 4, we can conclude that, higher maximum power constraint leads to higher utility, which in return proves the higher fairness controlled by SDN controller.

\section{CONCLUSions}

In this paper, we introduce one architecture with SDN controller for energy efficient resource allocation among different NSPs by considering heterogeneous QoS requirements. In order to take the competitive and fairness into account, the heterogeneous EE-RA problem is formulated as a reverse combinatorial auction game. Since the fractional WDP is NP hard, we propose an IAPA algorithm with transforming fractional objective into subtractive form, which is also demonstrated to be strategy-proof and with low computing complexity. Simulation results illustrate that, with SDN controller, the proposed algorithm convergences fast and can offer high fairness allocation among NSPs. Moreover, it is also proved to be robust with QoS requirement changing. We are going to improve these results by implementing primal-dual auction algorithm, simulating it more realistically with software, e.g., NS-3, and further applying it with real SDN controller.

\section{ACKNOWLEDGMENT}

The authors would like to thank Chinese Scholarship Council and FinCEAL Plus Travel Grant for supporting.

\section{REFERENCES}

[1] M. Arslan, K. Sundaresan, and S. Rangarajan, "Software-defined networking in cellular radio access networks: potential and challenges," IEEE Communications Magazine, vol. 53, no. 1, pp. 150-156, Jan. 2015.

[2] H. Ali-Ahmad and Cicconetti, "Crowd: An sdn approach for densenets," in 2013 IEEE Workshop on EWSDN. Berlin, 2013, pp. 25-31.

[3] H. Ali-Ahmad, C. Cicconetti, and D. L. Oliva., "An sdn-based network architecture for extremely dense wireless networks," in 2013 IEEE SDN4FNS, Trento, Nov 2013, pp. 1-7.

[4] E. Chavarria Reyes, I. Akyildiz, and E. Fadel, "Energy consumption analysis and minimization in multi-layer heterogeneous wireless systems," IEEE Tran. on Mobile Computing, vol. PP, no. 99, 2015.

[5] C. Liang and F. Yu, "Wireless network virtualization: A survey, some research issues and challenges," IEEE Communications Surveys Tutorials, vol. 17, no. 1, pp. 358-380, Firstquarter 2015.

[6] J. Tang and D. So, "Resource allocation for energy efficiency optimization in heterogeneous networks," IEEE Journal on Selected Areas in Communications, vol. PP, no. 99, May 2015.

[7] S. Jin and X. Zhang, "Optimal energy efficient scheme for mimo-based cognitive radio networks with antenna selection," in Proceedings of 2015 CISS, Baltimore, MD, March 2015, pp. 1-6.

[8] M. Peng and K. Zhang, "Energy-efficient resource assignment and power allocation in heterogeneous cloud radio access networks," IEEE Tran. on Vehicular Technology, vol. PP, no. 99, 2014.

[9] C. Yi and J. Cai, "Multi-item spectrum auction for recall-based cognitive radio networks with multiple heterogeneous secondary users," IEEE Tran. on Vehicular Technology, vol. 64, no. 2, pp. 781-792, Feb 2015.

[10] Y. Zhang, C. Lee, D. Niyato, and P. Wang, "Auction approaches for resource allocation in wireless systems: A survey," IEEE Communications Surveys Tutorials, vol. 15, no. 3, pp. 1020-1041, Third 2013.

[11] F. Martignon, S. Paris, I. Filippini, L. Chen, and A. Capone, "Efficient and truthful bandwidth allocation in wireless mesh community networks," IEEE/ACM Tran. on Networking, vol. 23, no. 1, pp. 161174, Feb 2015.

[12] C. Yi and J. Cai, "Two-stage spectrum sharing with combinatorial auction and stackelberg game in recall-based cognitive radio networks," IEEE Tran. on Communications, vol. 62, no. 11, pp. 3740-3752, 2014

[13] H. Al-Tous and I. Barhumi, "Resource allocation for multiple-user afofdma systems using the auction framework," IEEE Tran. on Wireless Communications, vol. 14, no. 5, pp. 2377-2393, May 2015.

[14] W. Dinkelbach, "On nonlinear fractional programming," Management Science, vol. 13, no. 7, pp. 492-498, 1967.

[15] S. Paris, F. Martignon, I. Filippini, and L. Chen, "An efficient auctionbased mechanism for mobile data offloading," IEEE Tran. on Mobile Computing, vol. 14, no. 8, pp. 1573-1586, Oct 2014. 